\title{
Comparative Study Between Classical Controllers and Inverse Dead Zone Control for Position Control of a Permanent Magnet DC Motor with Dead zone
}

\author{
C.A PEREZ-GOMEZ \\ MCIE \\ Universidad Autónoma \\ Metropolitana-Azcapotzalco \\ CDMX \\ MEXICO
}

\author{
J.U. LICEAGA-CASTRO \\ Departamento de Electrónica \\ Universidad Autónoma \\ Metropolitana-Azcapotzalco \\ CDMX \\ MEXICO
}

\author{
I.I. SILLER-ALCALA \\ Departamento de Electrónica \\ Universidad Autónoma \\ Metropolitana-Azcapotzalco \\ CDMX \\ MEXICO
}

Abstract: - In this work, a comparative study of three control strategies for the position control of a permanent magnet DC motor with dead zone is presented. The strategies analyzed are the classical PI controller, a new approach based on a linear controller with double integral effect, and the Inverse Dead Zone approach. Through the results here exposed it is shown that the new approach based on a controller with double integral effect results in a control system capable of achieving smaller position error, reducing the undesirable stick/slip effect without inducing high frequency oscillations or chattering in the control variable. In addition, and thanks to its linear nature, it is possible to determine stability and robustness of the resulting control system by means of the classical margins of gain and phase making this approach suitable for an engineering context

Key-Words: - PID controllers, Inverse Dead Zone, Position control, DC motor, Dead zone, Friction, Linear control.

Received: March 11, 2020. Revised: September 5, 2020. Re-revised: September 17, 2020. Accepted: September 27, 2020. Published: September 29, 2020.

\section{Introduction}

Permanent Magnet DC Motors (PMDC) are widely used for control purposes due to their high torque and wide speed ranges and because they are also relatively easy to analyze and model. These electromechanical devices are very common in applications where high power and precision are required, such as: biomedical equipment, medical robots, household appliances, machine tools and as an actuator in many industrial processes, [1], [2].

Despite the fact that PMDC motors are relatively easy to control, [3]; in conditions where it is necessary to operate at low speeds or where high precision position control is required, the design of the controller is not an easy task due to inherent non-linear motor dynamics, such as friction and the so-called dead zone, [4]-[6].

Usually, the dead zone is created by friction and inertias, especially by Coulomb friction force, [7][9]. This phenomenon is found in a wide variety of actuators such as electric motors and hydraulic valves, [10], [11]. In fact, it is considered one of the main nonlinearities in industrial processes, [12]. Because the dead zone can affect the performance and stability of position control systems, [13]-[15], several controllers have been proposed to try to reduce this problem, among the most used are those based on adaptative control, [16]-[21], sliding mode control [22], [23], and intelligent controllers, [24][27]. Although these strategies report excellent results, their conclusions are based on digital simulations and not by real-time implementations. This may be because the resulting controllers are highly complex, so their implementation becomes extremely difficult or almost impossible. Furthermore, most of these controllers use the socalled "Inverse Dead Zone" (IDZ) to cancel the dead zone phenomenon compromising the integrity of the process since the use of this strategy induces extremely high frequencies responses or chattering in the control variable, [28]. Another feature of most of these controllers is that they are designed for speed control purposes, where the dead zone no 
longer exists, and only a few have been designed for position control.

On the other hand, few controllers have been actually implemented to deal with the dead zone problem and its effects reporting adequate performance. For example, the predictive controller implemented in a plant whose actuators are DC motors, [29], or the switched controller proposed in [30], where the stick/slip phenomenon, caused by the dead zone and friction present in a magnetic levitation system, is reduced. Nevertheless, these controllers have the disadvantage that their design and real time implementation are usually complex. Furthermore, establishing necessary stability conditions is not straightforward.

In this work, a new strategy based on a linear controller with double integral and a lead compensator (PII) is proposed to reduce the dead zone phenomenon in the position control of a PMDC motor. This results in a controller that is easy to implement and, due to its linear nature, determine stability and robustness conditions using the classic phase and gain margins. The main objective behind the PII controller is to get the motor out of the dead zone as fast as possible, where it operates in open loop, to maintain or recover control over the motor.

In order to evaluate the performance of PII controller, the position response obtained by the PII controller is compared, through real-time implementation, with the position response of a simple PI controller and, by a digital simulation, with the response of a PI controller using inverse dead zone strategy. It is necessary to clarify that Inverse Dead Zone approach had to be digitally simulated -using a validated nonlinear model of the permanent magnet DC motor that includes dead zone and viscous and Coulomb frictions, [31]- to maintain integrity of the motor experiment bench which can be seriously damage by the high frequency modes or chattering in the control variable.

The article is structured as follows: in section 2, model of the PMDC motor of the Quanser Direct Current Motor Control Trainer (DCMCT) system is described. Section 3 presents the parameters and characteristics of the Quanser DCMCT system. Section 4 describes the design of the proposed PII controller. Section 5 shows the design of the PI controller. In Section 6, shows the design and digital simulation of the Inverse Dead Zone control approach. Section 7 describes the real time implementation of PII and PI controllers. In Section 8 an analysis of the results is presented. Finally, conclusions are shown in section 9 .

\section{Mathematical Modeling}

The PMDC motor can be modelled using two linear equations: one for electrical and the second for mechanical subsystems.

$$
\begin{gathered}
v(t)=R i(t)+L \frac{d i(t)}{d t}+E_{a} \\
T_{m}(t)=k_{m} i(t) \\
T_{m}(t)=J \frac{d^{2} \theta(t)}{d t^{2}}+b \frac{d \theta}{d t}
\end{gathered}
$$

Equation (1) represents the electrical subsystem where $v(t)$ is the applied armature voltage, $i(t)$ is the armature current, $E_{a}=k_{m} \frac{d}{d t} \theta(t)$ is the counter electromotive force and, $L$ and $R$ represent the inductance and resistance of armature winding, respectively.

The expression (3) represents the mechanical subsystem where $T_{m}(t)$ is the magnetic torque, $k_{m}$ is the motor constant, $J$ is the motors rotor equivalent moment of inertia, $b$ is the friction coefficient and $\theta$ is the angular position of the rotor. In this model the counter electromotive force present in the motor is assumed negligible.

The transfer function $G_{e}(s)$ of the electrical subsystem neglecting the counter electromotive force, since it is very small at low speed, results in:

$$
G_{e}(s)=\frac{I(s)}{V(s)}=\frac{\frac{1}{R}}{\left(\frac{L}{R} s+1\right)}
$$

Expression (5) shows the transfer function for the mechanical subsystem with $\omega(t)=\dot{\theta}$ as the rotor velocity.

$$
\begin{gathered}
G_{m}(s)=\frac{\omega(s)}{T_{m}(s)}=\frac{\frac{1}{b}}{\left(\frac{J}{b} s+1\right)} ; \\
T_{m}(s)=k_{m} I(s)
\end{gathered}
$$

Therefore, the transfer function relating rotors velocity $\omega(t)$ to input voltaje $v(t)$ is given by: 


$$
\frac{\omega(s)}{V(s)}=\frac{\frac{k_{m}}{R b}}{\left(\frac{J}{b} s+1\right)\left(\frac{L}{R} s+1\right)}
$$

Transfer function (6) can be simplified by pole dominance because the electrical mode is faster than the mechanical mode, [4], [32]; thus, the PMDC motor transfer function reduces to:

$$
\frac{\omega(s)}{V(s)}=\frac{\frac{k_{m}}{R b}}{\left(\frac{J}{b} s+1\right)}
$$

\section{Quanser's DCMCT system}

The DCMCT system is shown in figure 1, and the most important hardware components of this system are, [33]:

- An analog current sensor, which consists of a load resistor connected in series to the output of the amplifier.

- An analog speed sensor, whose range is $\pm 5 \mathrm{~V}$, so the signal obtained is scaled.

- A potentiometer which can be coupled via belt to the motor shaft.

- A quadrature optical encoder mounted to the rear of the motor.

- High quality Maxon's graphite brush coreless PMDC motor driven by a linear power amplifier.

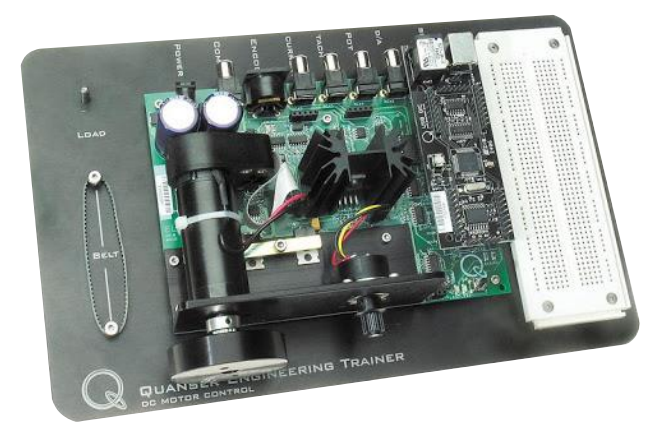

Fig. 1. Direct Current Motor Control Trainer system.

Although Maxon PMCD motor manufacturer parameters are available, table I, it is well known that these depend on the conditions under which they were measured and that they can also be altered by connection and operating conditions. Therefore, the parameters reported in [31], table II, will be used to model the PMDC motor.
Table I. Parameters Estimated by Experimental Tests

\begin{tabular}{|c|c|}
\hline Parameter & Value \\
\hline$R$ & $2.3724 \Omega$ \\
\hline$L$ & $17.7933 \times 10^{-3} \mathrm{H}$ \\
\hline$k_{m}$ & $50.2 \times 10^{-3} \mathrm{Nm} / \mathrm{A}$ \\
\hline$J$ & $3.10442 \times 10^{-3} \mathrm{~kg} \mathrm{~m}^{2}$ \\
\hline$b$ & $0.0314 \mathrm{~N} \mathrm{~m}$ \\
\hline
\end{tabular}

\section{PII Controller Design}

By the expression (7) and the parameters estimated of the table 2, the transfer function motor is shown in (8).

$$
G_{m}=\frac{0.545287}{(0.08 s+1)}
$$

Therefore, the transfer function that relates the position of the rotor and the supply voltage is shown in (9)

$$
G_{m_{\theta}}(s)=\frac{0.545287}{s(0.08 s+1)}
$$

Because PII controller will be discrete time implemented, the discretized transfer function of (9), including $\mathrm{ZOH}$ (Zero Order Hold), assuming a sampler period $T=0.02 \mathrm{~s}$ results in:

$$
\mathrm{G}_{\mathrm{m}_{\theta}}(\mathrm{z})=\frac{1.256 \times 10^{-3} \mathrm{z}+1.156 \times 10^{-3}}{\mathrm{z}^{2}-1.779 \mathrm{z}+0.7788}
$$

As mention above, the objective is to design a position controller easy to implement, analyze and capable of reducing the effects of the dead zone that, in conjunction with Coulomb and viscous frictions, affects control system performance and possibility of achieving zero position error; that is, the so call Stick-Slip effect. Therefore, through PII controller is possible to attenuate response oscillations -limit cycle- rendering small position errors.

The controller design approach is based on the Root Locus approach since it allows to easily determine the location of controller poles and zeros. In the same way, through this method it is possible to secure control system stability. 


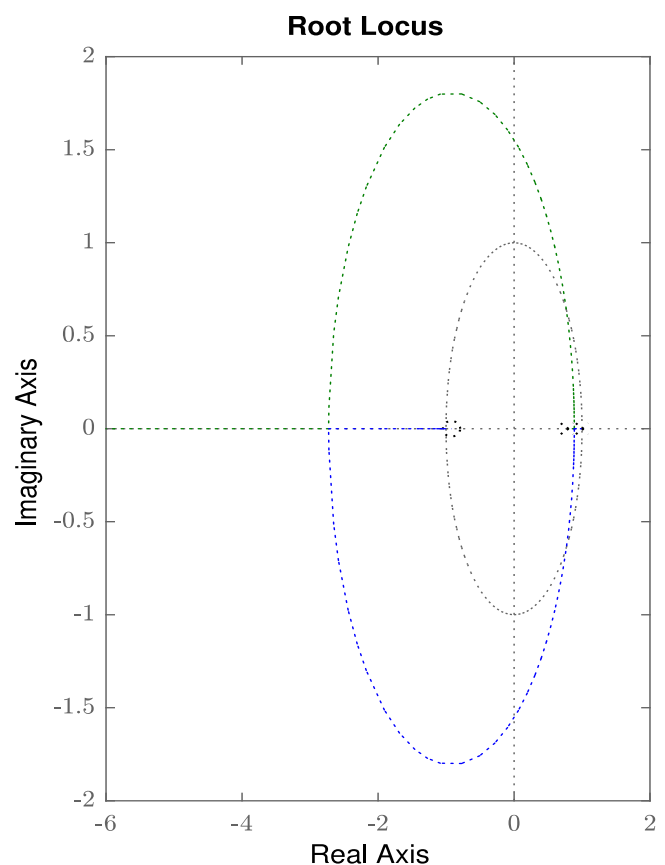

Fig. 2. Root Locus plot of the PMDC motor.

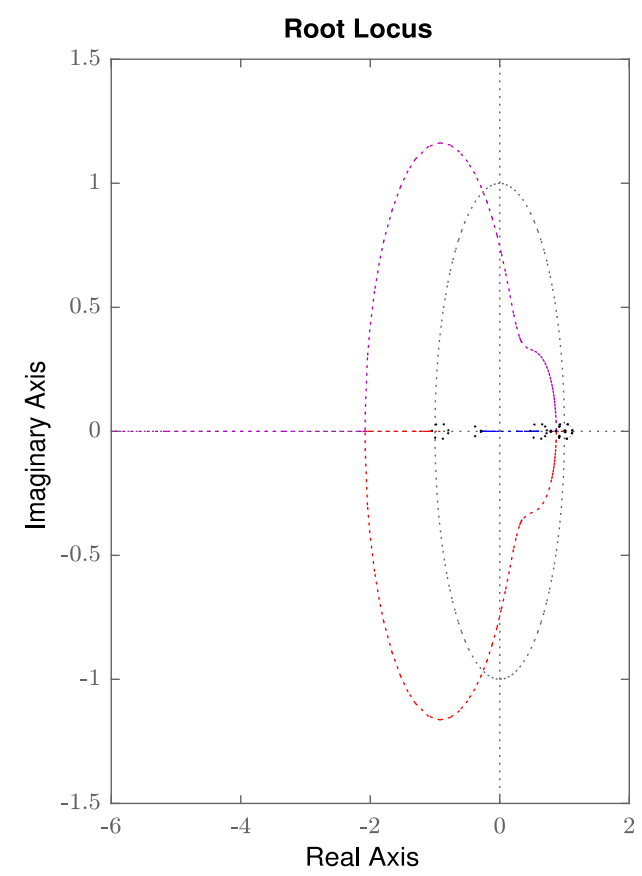

Fig. 3. Root Locus plot of the PMDC motor with PII controller.

The root locus plot for the PMDC motor is shown in 2, corresponding to the transfer function (10). The figure shows that the system is critically stable. It has two poles: one at 1 , due to the integral effect of the plant; and the second at 0.7788 . It also has a single zero located at -0.92 , very close to the unit circle.
Considering that the proposed controller has two integrators, the system will have three poles at $(1,0)$, on the unit circle. Thus, the lead compensator and the zeros corresponding to each of the integrators, must be positioned ensuring system stability and robustness.

The root locus strategy for controller's design is based on the fact that closed loop poles trajectories starts at the open loop poles and end at the open loop zeros. Therefore, it is possible to ubicate controller poles and zeros assuring close loop stability, remaining only to calculate controllers gain to assure stability and performance.

The resulting PII controller is given by:

$$
C(z)=\frac{80 z^{3}-207.2 z^{2}+174.7 z-47.52}{z^{3}-1.7 z^{2}+0.4 z+0.3}
$$

Figure 3 shows the root locus of the PMDC motor with the PII controller. In the figure can be observed that the system is stable.

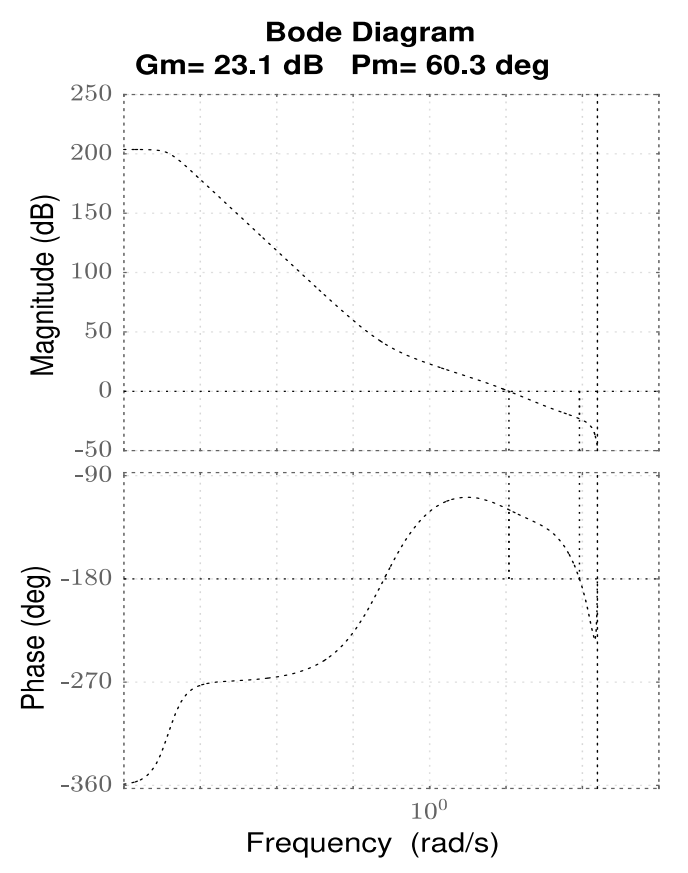

Fig. 4. Control system using PII controller Bode diagram.

Also, figure 4 shows the Bode diagram of the control system $C(z) G_{m_{\theta}}(z)$, where it is possible to observe and determine that the system has a phase and gain margins $M_{f}=60.3^{\circ}$ and $M_{g}=23.1 \mathrm{dBs}$, respectively, with a bandwidth $\omega_{B}=10.9 \mathrm{rad} / \mathrm{s}$. It is possible to assure stability and robustness based on phase and gain margins as these indicators are valid when the input voltage induces a magnetic torque out of the dead zone, that is, when the motor operates out of the dead zone and is under control. 
On the other hand, when the motor works within the dead zone it is operating open loop and because it is stable, global stability can be assured.

\section{PI Controller Design}

To compare the PII controller performance a PI controller is designed. The resulting PI controller is designed like the PII controller resulting in:

$$
C(z)_{P I}=\frac{5(2 z-1.98)}{z-1}
$$

Figure 5 shows the root locus plot corresponding the control system $C(z)_{P I} G_{m_{\theta}}$. Analyzing the figure, is possible to determine that the system is stable. Due to the integral effect one pole is located on the unit circle, while the zero is located at 0.99 . The zero observed in the negative values of the unite circle belongs to the PMDC motor.

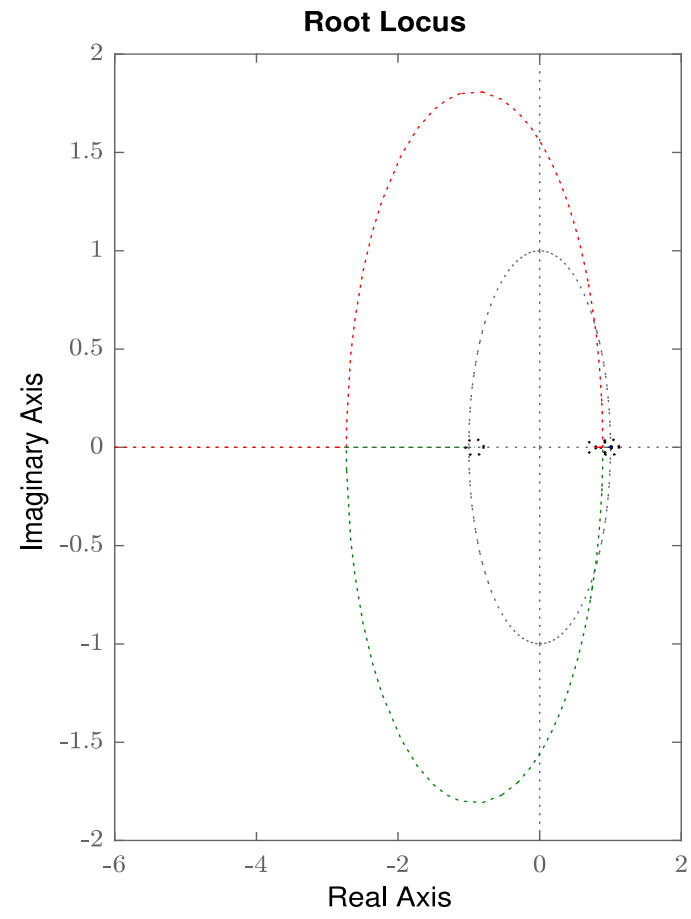

Fig. 5. Root Locus plot of the PMDC motor with PI controller.

The Bode diagram of the control system $C(z)_{P I} G_{m_{\theta}}$ is shown in figure 6. The control system has a gain margin of $M_{g}=25.3 \mathrm{dBs}$ with a bandwith of $\omega_{B}=33.9 \mathrm{rad} / \mathrm{s}$ and a phase margin of $M_{f}=59.4^{\circ}$, therefore the system is robust.

\section{Inverse Dead zone Design}

IDZ strategy allows canceling the dead zone phenomenon and, together with a position controller, reduces steady state error and stick/slip effect caused by the dead zone in conjunction with the Coulomb and viscous frictions.

The symmetric inverse dead zone model is shown in figure 7 , where $u_{d}$ is the desired output, $u$ is the input voltage in the inverse dead zone, which is the same as the voltage supplied by the controller. $\delta_{v}$ is the dead zone breakdown voltage.

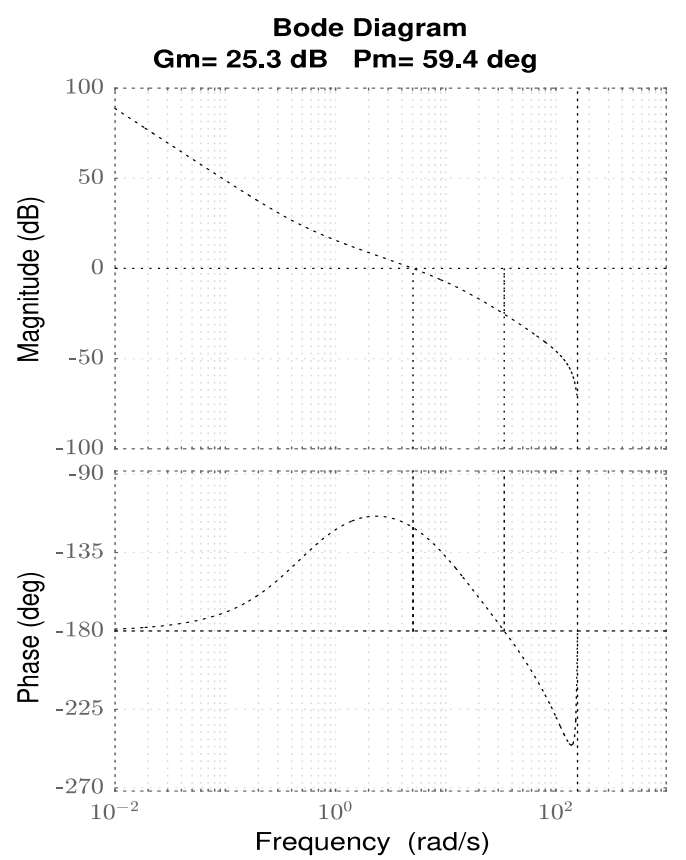

Fig. 6. Control system using PI controller Bode diagram.

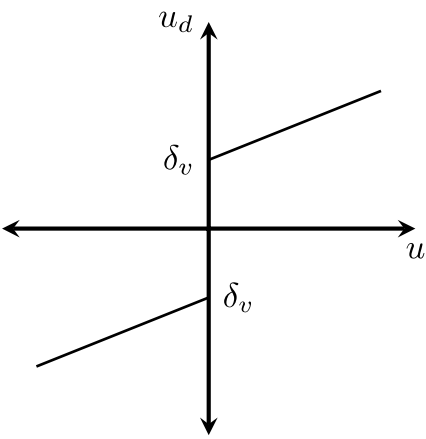

Fig. 7. Inverse dead zone symmetrical model.

The inverse dead zone is modeled by the block function in Matlab ${ }^{\circledR} /$ Simulink $^{\mathrm{TM}}$. The code that model this strategy is shown in figure 8 , where $u d$ represents the desired output, $u$ is the inverse dead zone input and $d v$, represents the breaking point of the dead zone. Inverse dead zone parameters 
depend on dead zone parameters. This were obtained from [31].

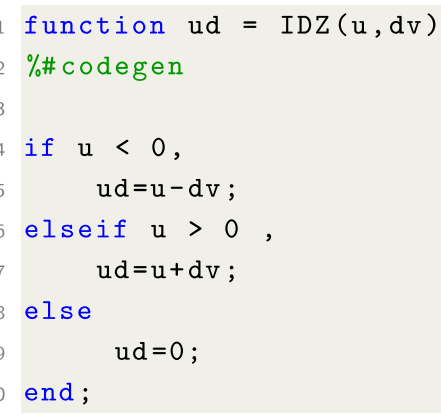

Fig. 8. Inverse dead zone Matlab ${ }^{\circledR} /$ Simulink $^{\mathrm{TM}}$ code.

\section{Implementation}

The PII and PI controllers are implemented using the Matlab ${ }^{\circledR} /$ Simulink $^{\mathrm{TM}}$ Real-Time tool. The data acquisition is carried out through the National Instrument (NI) SCB-68 shielded I/O connector block, which has 68 screw terminals. This block is connected to the DAQ I/O device PCIe-6363 also designed by NI. The PCIe-6363 has 32 16-bit analogic channels, with a maximum sampling rate of the 2MS/s and supported voltage of the input channels is from $+0.1 \mathrm{~V}$ to $+10 \mathrm{~V}$.

The control system based on PII controller and the PI controller is tested in the DCMCT system assuming a sequence of square inputs as reference signal. The dead zone of the DCMCT system is found in the range of $-0.35 \mathrm{~V}$ to $0.3 \mathrm{~V}$ for the input voltage. That is, the dead zone is not symmetrical.

Likewise, both drivers are compared using the non-linear model of the Maxon PMDC motor, which includes the DCMCT system, proposed in [31].

Figure 9 shows the PII controller rotor position response. From this figure is possible to observe that the controller has a good performance with very low steady state error of approximately $|e|<0.004$.

The low frequency oscillations of the control variable are due to the high gain generated by the double integrator necessary to get the process out of the dead zone as fast as possible. Furthermore, PII controller high gain can adequately compensate for the noise produced by the sensor.

Figure 10 shows the response of the rotor position to variations of the reference signal from $0.5 \mathrm{~V}$ to $0 \mathrm{~V}$ and from $0 \mathrm{~V}$ to $-0.5 \mathrm{~V}$. From this figure it is possible to observe that the performance of the controller when the motor rotates counterclockwise is the same as that of clockwise rotation despite the non-symmetrical dead zone.

In both figures it can be seen that the non-linear model and the real motor have similar results, the variation in the responses, is due to the fact that the sensor noise and other dynamics present in the system are not considered in the non-linear model. Despite this, it is possible to observe that the nonlinear model is a good representation of the real system.

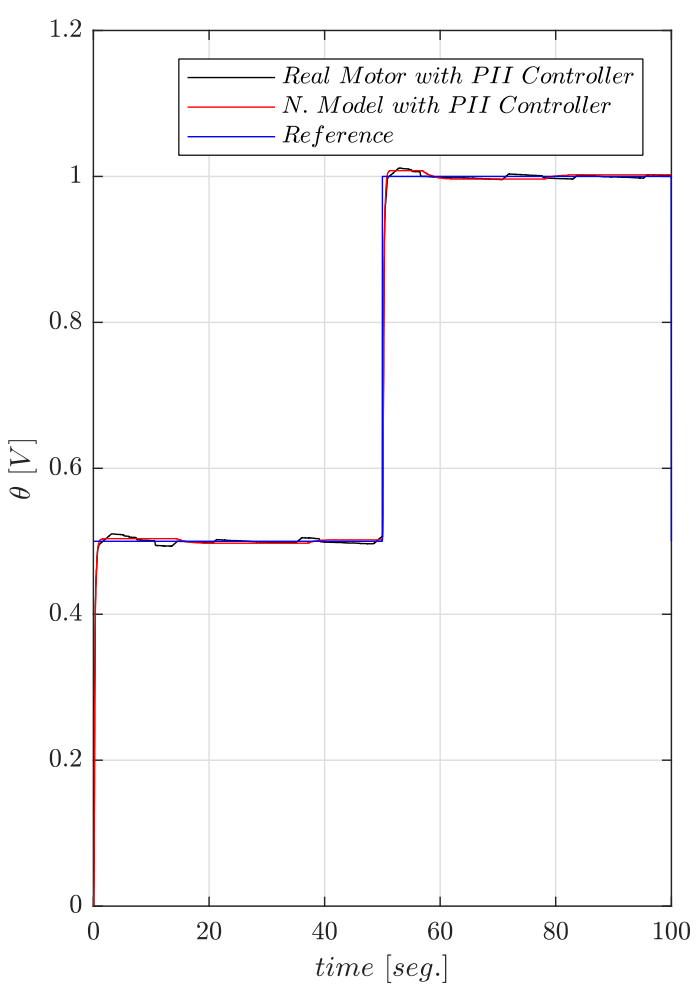

Fig. 9. Motor position step responses with PII controller.

The rotor position responses of the PI controller are shown in figures 11 and 12. Through both figures it is possible to observe that the PI controller, despite having a good performance in the real system, cannot be able to compensate the noise of the sensor with fast as the controller with double integral effect does. This is clearly shown in the error presented by the oscillations.

Likewise, figure 13 shows the comparison between PII and PI controllers under disturbances, where it is observed that the first responds more quickly and with better performance than the second. The response of the PI controller tends to be slower and in the event of consecutive disturbances 
the system would not be able to compensate for them.

The responses of the PI + IDZ controller are shown in figures 14 and 15 . This controller is implemented through the non-linear model because the response presents high frequency oscillations as shown in figure 14. These oscillations are due to the presence of chattering in the control signal as observed in figure 16, thus confirming what most studies have reported about the use of this strategy to reduce the dead zone phenomenon. Due to this, the analysis of this strategy is only carried out by simulation, where it is observed that this strategy has a good performance in the system with a constant low steady state error.

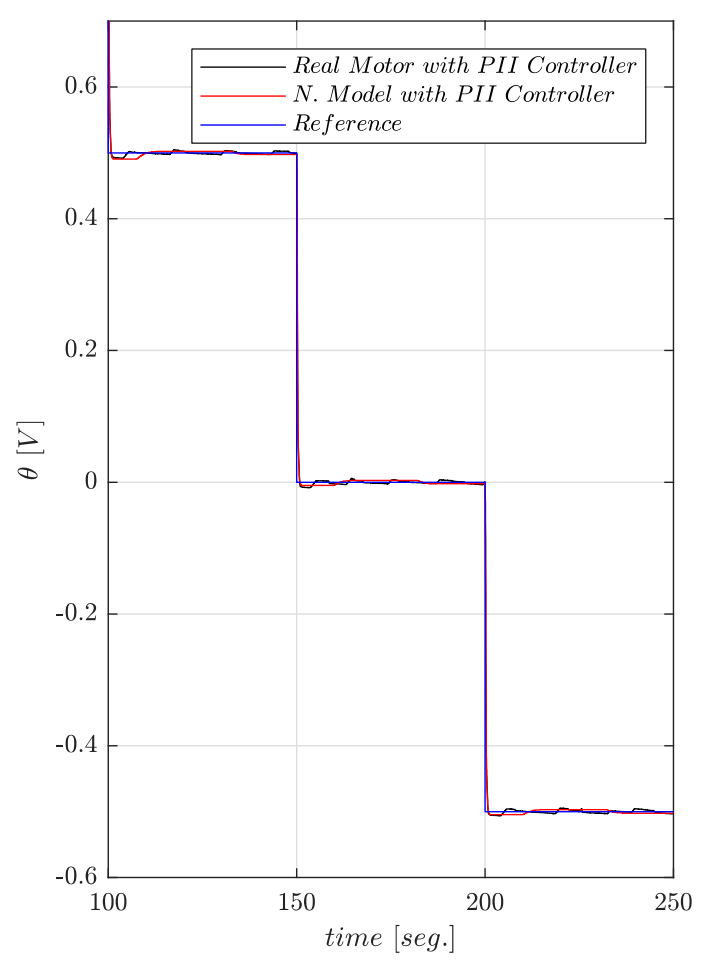

Fig. 10. Motor position step responses with PII controller.

\section{Analysis of Results}

Observing figures shown in section 7 , is clear that the objectives of the proposed PII controller are satisfied. The system has a small steady state position error, can compensate the sensor noise and is relatively easy to design and implement. Also, no dead zone cancellation is required, neither to know dead zone parameters whether symmetric or not. Likewise, through comparison among other controllers is clearly that the proposed strategy provides a better performance reducing dead zone effects.

Observing the PII controller output, shown in figure 17, the system does not present any type of chattering, normally present in approaches where IDZ is used. Similarly, due to the integral double effect presented by the PII controller, its output tends to be parabolic rather than linear, allowing the system to stay within the dead zone for the shortest possible time. Additionally, the controller with double integral effect and lead compensator allows to determine stability and robustness because it is based on design strategies of the classic control as shown in section 4 .

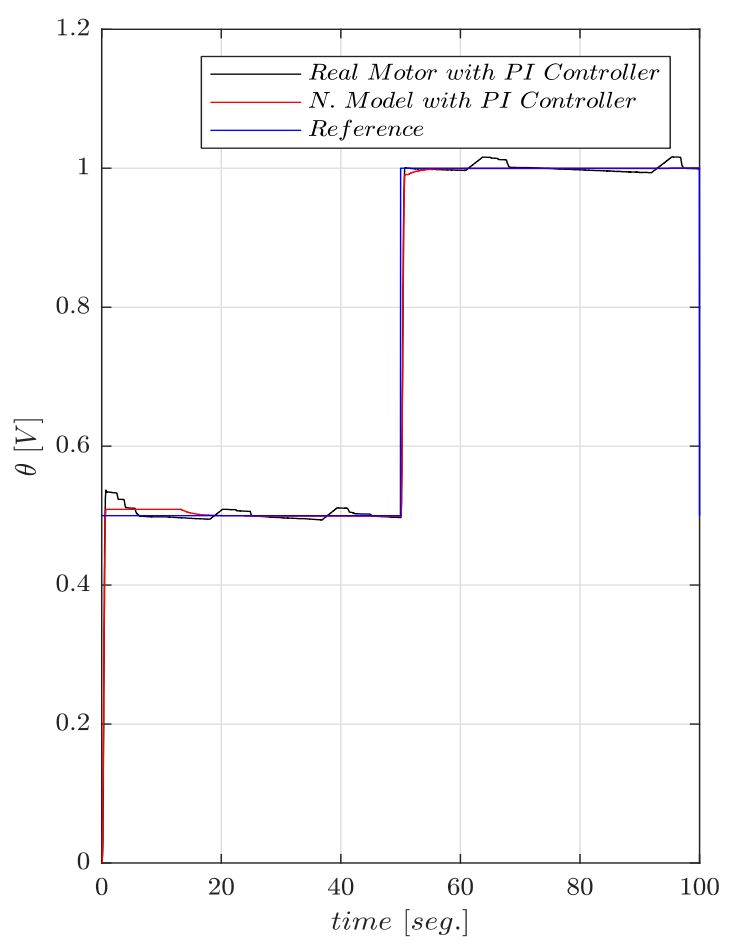

Fig. 11. Motor position step response with PI controller.

On the other hand, although classical PI controller is easy to implement and design, and with good responses, figure 11 , it cannot reduce the stick/slip phenomenon induced by the dead zone and friction, resulting in higher errors and oscillations than those obtained by PII controller making it not suitable for high precision control requirements. 


\section{Conclusion}

A new linear position control system was designed and implemented to reduce the dead zone phenomenon in a PMDC motor. The controller has a double integral effect and lead compensator. Through the implementation of the control system it was observed that it generates a very small steady state error and provides a good performance against the effects generated by the nonlinearities of the system and sensor noise.

Because it is based on classic control design strategies, it is possible to determine the stability and robustness of the system using classical control indicators. Moreover, because the PII controller forces the motor out of the dead zone as fast as possible it is not necessary to know the dead zone parameters and characteristics, resulting in a controller easy to implement and assess.

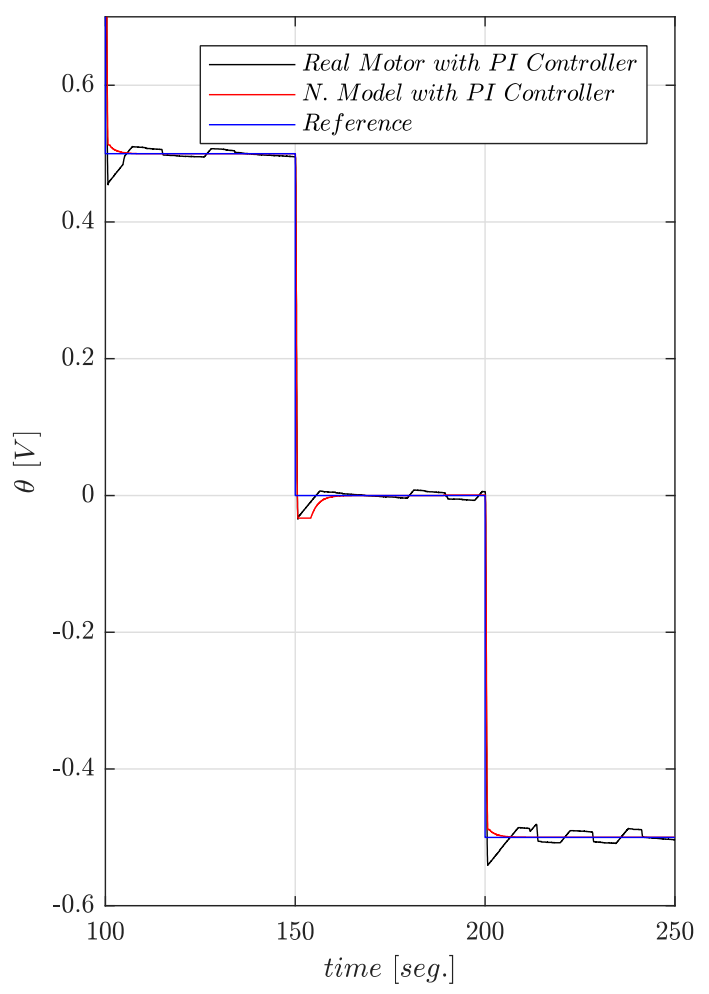

Fig. 12. Motor position step response with PI controller.

On the other hand, the PII controller was compared by real-time implementation with the PI controller, and by simulation with a PI+IDZ controller, where it was possible to determine that the strategy proposed provides a better behaviour against disturbances without presenting any type of chattering in the control signal.
Based on the results here presented, controllers with double integral action may represent an adequate alternative to the design of control systems for any kind of actuators, not only electric motors, subjected to the combined action of dead zone and Coulomb friction.

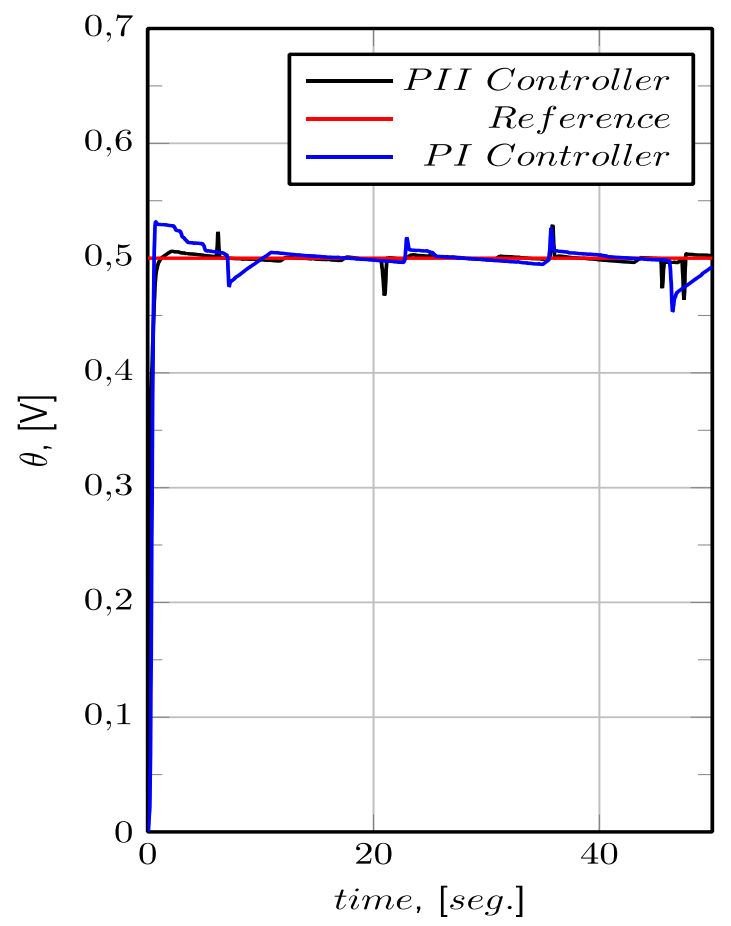

Fig. 13. Comparison between PI and PII controllers under disturbances.

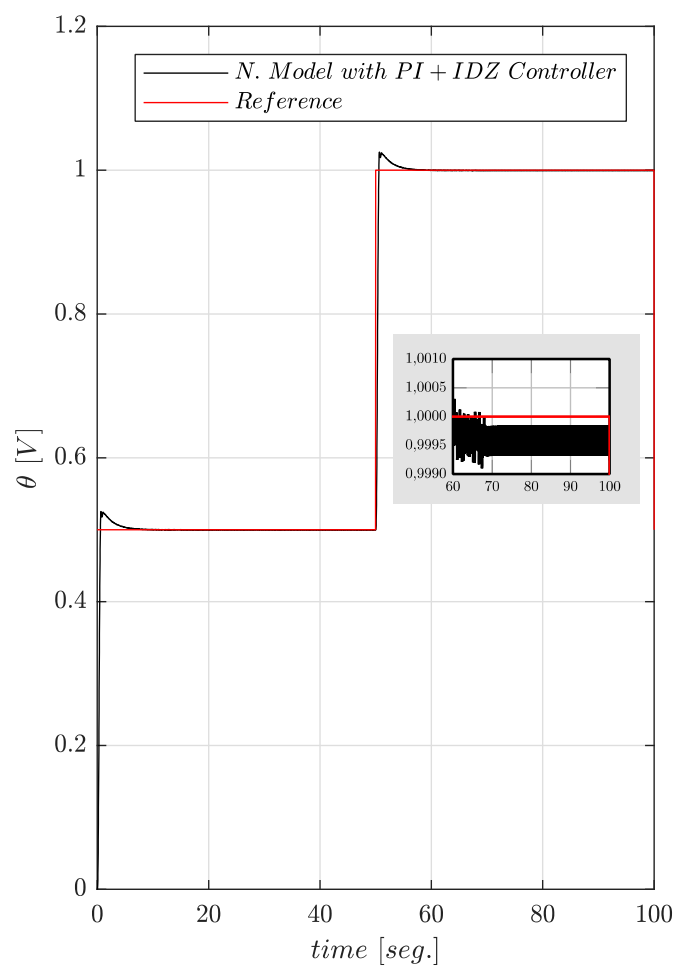


Fig. 14. Motor position step response with PI+IDZ controller.

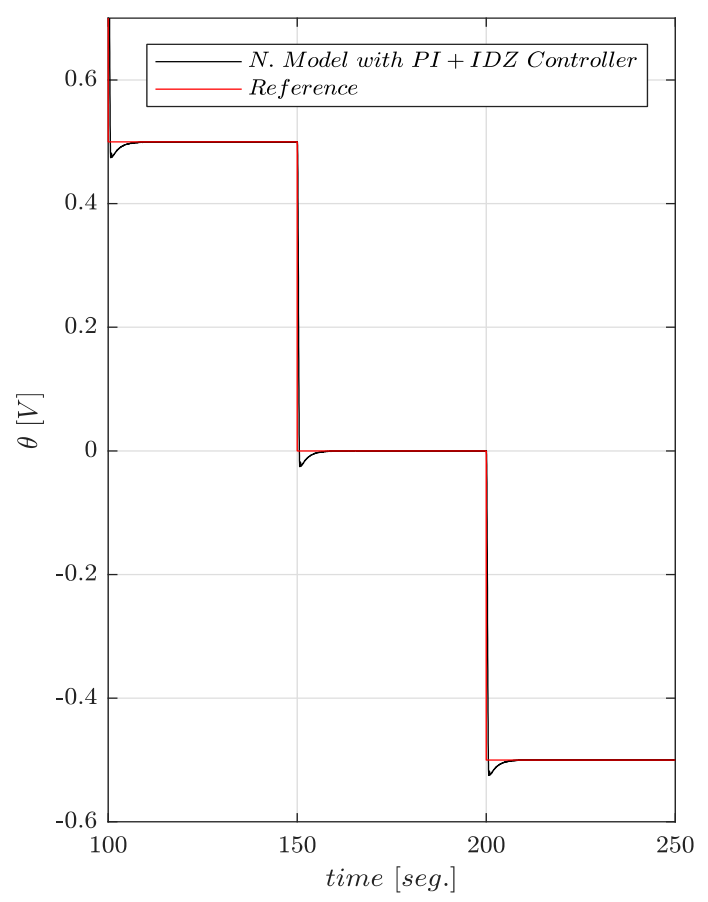

Fig. 15. Motor position step response with PI+IDZ controller.

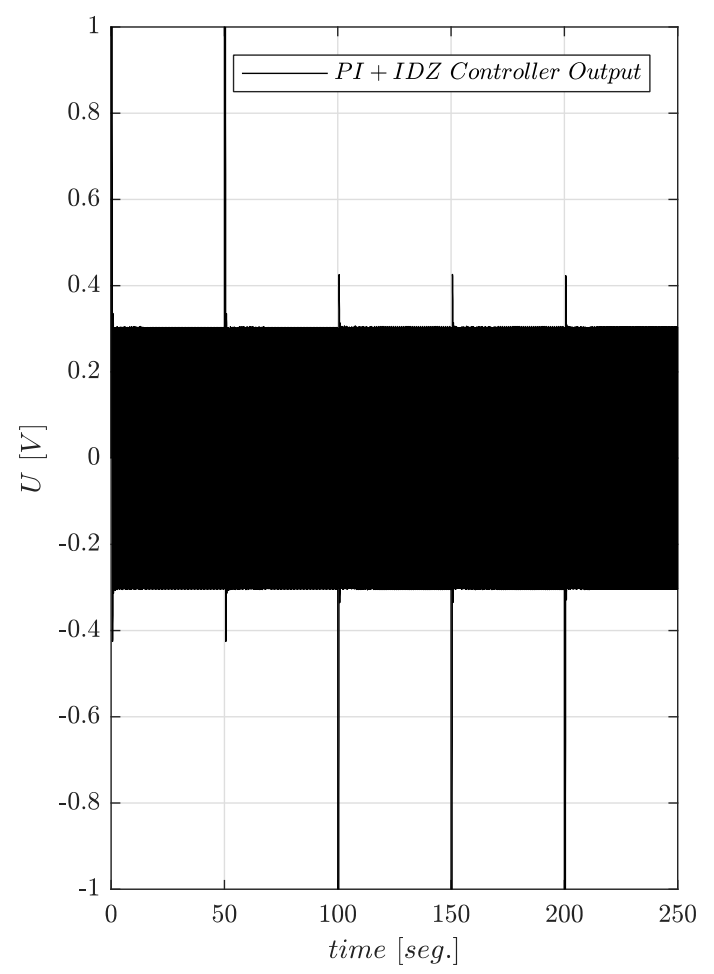

Fig. 16. PI+IDZ controller output.

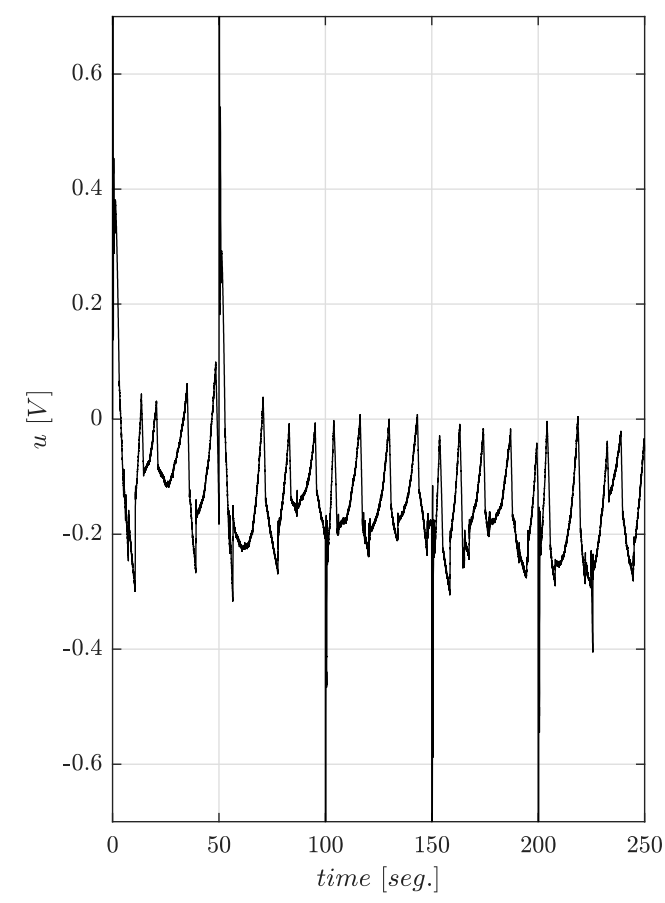

Fig. 17. PII Controller output.

\section{References:}

[1] J.-H. Horng, "Neural adaptive tracking control of a dc motor," Information sciences, vol. 118, no. 1-4, pp. 1-13, 1999.

[2] K. Saurav and R. Potluri, "Sensorless speed control of a permanent magnet dc motor by compensating the plant nonlinearities," in 2013 IEEE International Symposium on Industrial Electronics. IEEE, 2013, pp. 1-4.

[3] M. Charles, R. Okoro, I. Ikposhi, and D. Oku, "Reliable control of pmdc motor speed using matlab," International Journal of Scientific \& Engineering Research, vol. 6, no. 12, pp. 208216, 2015.

[4] H. Chu, W. Tao, B. Gao, Q. Liu, and H. Chen, "Speed control of the permanent-magnet dc motor subjected to uncertainty and disturbance," in 2016 35th Chinese Control Conference (CCC). IEEE, 2016, pp. 46644669.

[5] N. P. Mahajan and S. Deshpande, "Study of nonlinear behavior of dc motor using modeling and simulation," International Journal of Scientific and Research Publications, vol. 3, no. 3, pp. 576-580, 2013.

[6] S. Syukriyadin, S. Syahrizal, G. Mansur, and H. Ramadhan, "Permanent magnet dc motor control by using arduino and motor drive module bts7960," in IOP Conference Series: 
Materials Science and Engineering, vol. 352, no. 1. IOP Publishing, 2018, p. 012023.

[7] M. F. Borowski, "Analysis and control of a dc electric drive exhibiting dead zone characteristics," University of New Hampshire, Department of Electrical and computer Engineering.

[8] D. Recker, P. Kokotovic, D. Rhode, and J. Winkelman, "Adaptive nonlinear control of systems containing a deadzone," in [1991] Proceedings of the 30th IEEE Conference on Decision and Control. IEEE, 1991, pp. 21112115.

[9] J. Peng and R. Dubay, "Identification and adaptive neural network control of a dc motor system with dead-zone characteristics," ISA transactions, vol. 50, no. 4, pp. 588-598, 2011.

[10] N. J. Ahmad, H. K. Ebraheem, M. J. Alnaser, and J. M. Alostath, "Adaptive control of a dc motor with uncertain deadzone nonlinearity at the input," in 2011 Chinese Control and Decision Conference (CCDC). IEEE, 2011, pp. 4295-4299.

[11] X. Su, Z. Liu, G. Lai, C. L. Philip Chen, and C. Chen, "Direct adaptive compensation for actuator failures and dead-zone constraints in tracking control of uncertain nonlinear systems," Information Sciences, ELSEVIER, pp. 1-12, 2017.

[12] J. d. J. Rubio, Z. Zamudio, J. Pacheco, and D. Mujica Vargas, "Proportional derivative control with inverse dead-zone for pendulum systems," Mathematical Problems in Engineering, vol. 2013, 2013.

[13] C. Hu, B. Yao, and Q. Wang, "Adaptive robust precision motion control of systems with unknown input dead-zones: A case study with comparative experiments," IEEE Transactions on Industrial Electronics, vol. 58, no. 6, pp. 2454-2464, 2010.

[14] Q. Chen, L. Yu, and Y. Nan, "Finite-time tracking control for motor servo systems with unknown dead-zones," Journal of Systems Science and Complexity, vol. 26, no. 6, pp. 940-956, 2013.

[15] M. P. Aghababa, "Adaptive control for electromechanical systems considering deadzone phenomenon," Nonlinear Dynamics, vol. 75, no. 1-2, pp. 157-174, 2014.

[16] N. J. Ahmad, M. J. Alnaser, E. Sultan, and K. A. Alhendi, "Robust adaptive control for a class of systems with deadzone nonlinearity," Intelligent Control and Automation, vol. 6, no. 01, p. 10, 2015.
[17] N. J. Ahmad, E. K. Sultan, M. Q. Qasem, H. K. Ebraheem, and J. M. Alostad, "Adaptive control for a class of systems with output deadzone nonlinearity," Intelligent Control and Automation, vol. 6, no. 04, p. 215, 2015.

[18] J. Zhou, C. Wen, and Y. Zhang, "Adaptive output control of nonlinear systems with uncertain dead-zone nonlinearity," IEEE Transactions on Automatic Control, vol. 51, no. 3, pp. 504-511, 2006.

[19] X. Liu, W. Jiang, and X.-C. Dong, "Nonlinear adaptive control for dynamic and dead-zone uncertainties in robotic systems," International Journal of Control, Automation and Systems, vol. 15, no. 2, pp. 875-882, 2017.

[20] Y. Wang, C. Deng, and G. Den, "Adaptive controller design for the control systems with dead-zone," International Journal of Computer Applications, vol. 139, no. 6, pp. 29-33, 2016.

[21] J. Zhou and X. Shen, "Robust adaptive control of nonlinear uncertain plants with unknown dead-zone," IET Control Theory \& Applications, vol. 1, no. 1, pp. 25-32, 2007.

[22] W. M. Bessa, A. S. de Paula, and M. A. Savi, "Sliding mode control with adaptative fuzzy dead-zone compensation for uncertain chaotic systems," Springer, pp. 1989-2001, 2012.

[23] C.-F. Hsu, "Intelligent total sliding-mode control with dead-zone parameter modification for a dc motor driver," IET Control Theory \& Applications, vol. 8, no. 11, pp. 916-926, 2014.

[24] C.-C. Chiang, "Adaptive fuzzy tracking control for uncertain nonlinear time-delay systems with unknown dead-zone input," Mathematical Problems in Engineering, vol. 2013, 2013.

[25] J. Wang, "Adaptive fuzzy control of directcurrent motor dead-zone systems," Int. J. Innov. Comput. Inf. Control, vol. 10, no. 4, pp. 1391- 1399, 2014.

[26] L. Liu, Y.-J. Liu, and C. P. Chen, "Adaptive neural network control for a dc motor system with dead-zone," Nonlinear Dynamics, vol. 72, no. 1-2, pp. 141-147, 2013.

[27] J. O. Jang, "A deadzone compensator of a dc motor system using fuzzy logic control," IEEE Transactions on Systems, Man, and Cybernetics, Part C (Applications and Reviews), vol. 31, no. 1, pp. 42-48, 2001.

[28] J. D. Fortgang, L. E. George, and W. J. Book, "Practical implementation of a dead zone inverse on a hydraulic wrist," in ASME 2002 international mechanical engineering congress and exposition. American Society of Mechanical Engineers Digital Collection, 2002, pp. 149-155. 
[29] G. Galuppini, L. Magni, and D. Martino Raimondo, "Model predictive control of systems with deadzone and saturation," Control Engineering Practice, ELSEVIER, no. 78, pp. 56-64, 2018.

[30] D. H. Alcántara, L. A. Brooks, J. L. Castro, and E. L. Castro, "Control conmutado para un sistema de levitación magnética con atascamiento- deslizamiento," Revista Iberoamericana de Automática e Informática industrial, vol. 11, no. 3, pp. 285-294, 2014.

[31] A. Pérez, J. Liceaga, and I. Siller, "Non-linear modeling and identification of a permanent magnet dc motor," Proceedings of the 24th CSCC congress, 2020.

[32] F. MONASTERIO and A. Gutiérrez, "Modelo lineal de un motor de corriente continua," 2012.

[33] U. Guide, "Quanser engineering trainer dc motor control, quanser consulting."

\section{Contribution of individual authors to the creation of a scientific article}

Arturo C. Pérez-Gómez performed the simulations, ran the experiments, and contributed to the design of the controllers.

Jesús U. Liceaga-Castro and Irma I. Siller-Alcalá were the leaders and responsible of the project and contribute with the analysis and design of the control strategies.

\section{Sources of funding for research presented in a scientific article or scientific article itself}

The project was funded by the UAM-Azc and by CONACYT scholarship grant to Arturo C. PérezGómez.

\section{Creative Commons Attribution License 4.0 (Attribution 4.0 International, CC BY 4.0)}

This article is published under the terms of the Creative Commons Attribution License 4.0

https://creativecommons.org/licenses/by/4.0/deed.en_US 